\title{
Neglected Posterior Dislocation of Shoulder
}

\author{
Brig VP Pathania ${ }^{*}$,ss, Lt Col NC Arora*, Dr JBV Prasad ${ }^{*}$
}

MJAFI 2003; $59: 63-64$

Key Words : Neglected; Posterior shoulder dislocation

\section{Introduction}

$\mathrm{P}$ osterior dislocation of shoulder is quite an uncommon injury as compared to anterior dislocation [1], and it creates a diagnostic trap for the unwary surgeon [2]. The incidence is estimated to be at $2 \%$ but is difficult to ascertain because of the infrequency with which this diagnosis is made [3]. It is commonly associated with an impaction or "encoche" fracture of the humeral head which causes locking of the humeral head behind the glenoid [4]. In this position, it is often unrecognised and may not be given adequate treatment until some weeks after the injury [5]. Routine radiographs of the shoulder, are also misleading in arriving at the accurate diagnosis of posterior dislocation of shoulder. This report presents a case of neglected posterior shoulder dislocation treated by open reduction and transposition of subscapularis tendon into the humeral head defect.

\section{Case Report}

A 38 year old right hand dominant serving soldier sustained an electric shock injury to right upper limb from a domestic electric source of 230 volts. 15 amperes $\mathrm{AC}$ on $02 \mathrm{Dec} 99$. Following this, he developed pain and restriction of movement of right shoulder. After clinical and radiological evaluation he was treated as a case of "frozen shoulder" secondary to injury to supraspinatus tendon in a peripheral hospital. Patient had no relief of symptoms and was transferred to our hospital for evaluation of brachial plexus injury and an orthopaedic consultation was sought.

On examination he was found to have loss of normal deltoid contour of right shoulder, a prominent acromion, a palpable globular mass of humeral head posteriorly below the acromion. The coracoid process was prominent anteriorly and was easily palpable. All the movements of the right shoulder were restricted and the rotational movements were absent. There was no distal neurovascular deficit. Clinically posterior dislocation of shoulder was suspected and this was confirmed. on trans-axillary view radiograph and computerised tomography (CT) scan showed a large $(25-30 \%)$ impacted fracture on the anteromedial aspect of the humeral head (Reverse Hill-Sach's lesion) (Fig 1).

The patient was taken up for surgery on 3 Jan 2000. Shoulder joint was exposed by anterior Henry's deltopectoral approach. Head of the humerus was found to be dislocated behind the glenoid. There was a vertical impacted fracture on the anteromedial surface of the head. Open reduction was achieved. Subscapularis tendon was detached from its insertion and reattached into the impacted fracture defect on the humeral head (McLaughlin's procedure). The glenohumeral joint was transfixed with two K-wires.

Post-operatively the shoulder was maintained in adduction and slight internal rotation by strapping. The arm was held posterior to the mid sagittal line of the body. Sutures and K-wires were removed at two weeks and strapping continued for a further two weeks. Range of motion exercises were started at the end of four weeks. Postoperative CT scan and radiographs after eight weeks (Fig 2) showed a reduced gleno-humeral joint and partial healing of the humeral head fracture defect. Patient regained useful range of movements and was able to use his right upper limb for all his daily activities (Fig 3 ).

\section{Discussion}

Posterior shoulder dislocation is an infrequent injury caused due to trauma or violent contracture of the muscles of the shoulder girdle as in epilepsy or in electric shock injuries. Sir Astley Cooper [6] has reported this lesion in epileptics as early as 1855, and Thomas [7] has described this lesion due to electric shock in 1937. The humeral head, displaced posteriorly is pulled back

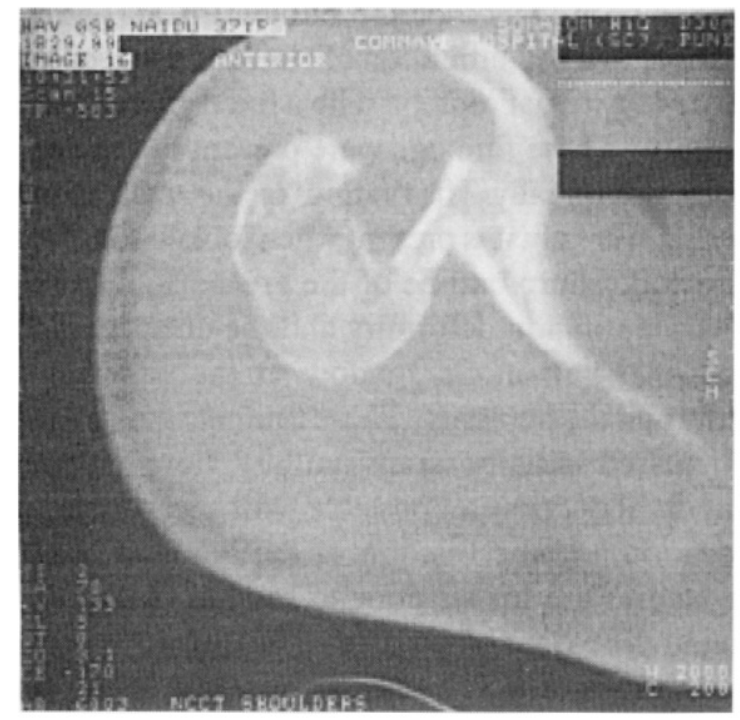

Fig. 1 : Pre-op CT scan showing the impacted fracture on dislocated humeral head

-Dy Commandant, Command Hospital (Westem Command), Chandimandir - 134 107. ${ }^{+}$Classified Specialist (Surgery and Orthopaedics). Base Hospital, Delhi Cantt - 110 10, Post Graduate Trainee in Orthopaedics. Armed Forces Medical College, Pune - 411040. 


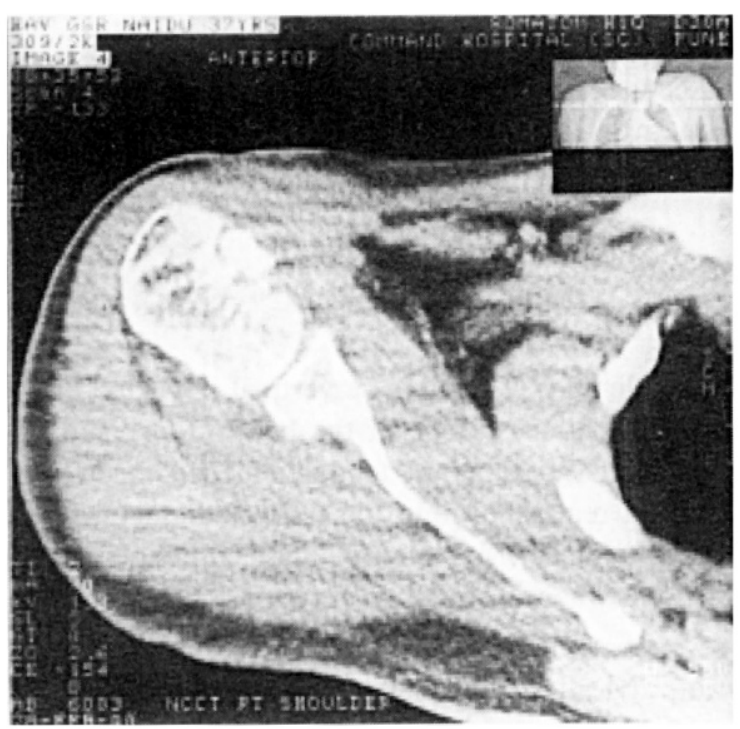

Fig. 2 : Post-op CT scan showing reduced humeral head and healing fracture

violently by the capsule and the anterior musculature of the shoulder. This causes impaction of the cancellous humeral head against the strong posterior rim of the glenoid, causing the characteristic "encoche" fracture or the vertical impacted fracture, described by Mclaughlin in 1959 [2].

Many such dislocations are missed by the surgeon because of the rarity of the lesion. Frequent cause of misdiagnosis is the failure to take adequate radiographs [8]. The physical findings, when looked for are characteristic and unequivocal. As described by Mclaughlin, they include an increased palpable prominence of the coracoid, decreased anterior prominence of humeral head, increased palpable posterior prominence of the humeral head below the acromion, marked limitation of abduction and complete absence of external rotation with a fixed internal rotation deformity. These findings were present in this case but were missed initially. The findings on the antero-posterior radiograph are always present when looked for carefully and include sharp outline of the greater tuberosity due to internal rotation deformity and the disappearance of the normal half-moon shadow of the superimposed glenoid and the humerus. These findings though present were missed initially. Trans-axillary view with arm in as much abduction as possible will clearly show the dislocation and the fracture of the humeral head. CT scan studies are far superior to routine radiographs in defining the extent of the displacement and the osseous abnormalities. The CT scan showed the lesion on the humeral head in our case. The treatment of neglected

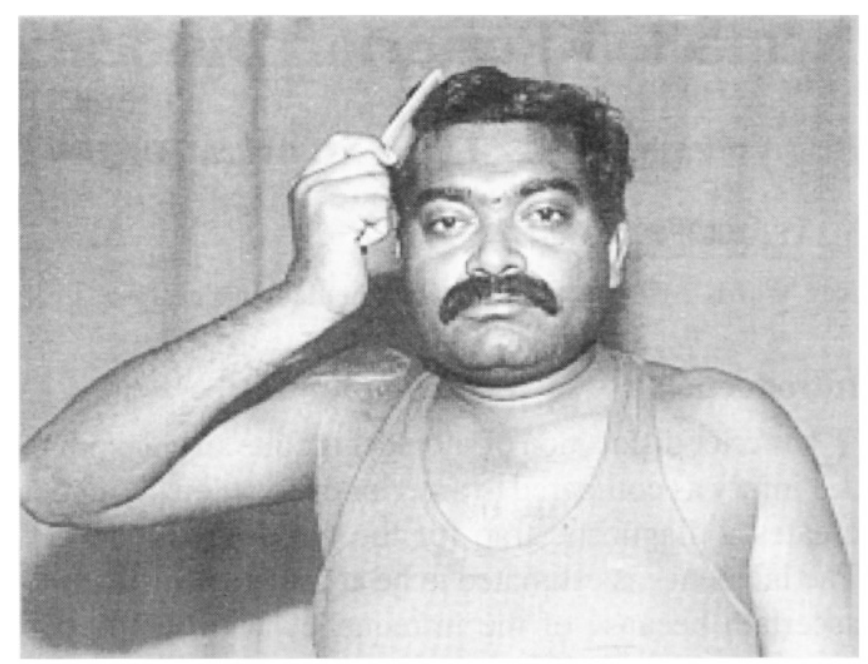

Fig. 3 : Clinical photograph of patient showing recovery after surgery

posterior shoulder dislocation is open reduction and transfer of attachment of subscapularis tendon into the humeral head defect (McLaughlin's procedure) or transfer of the tendon with a fragment of the lesser tuberosity (Neer's modification). When the fracture defect is more than $40 \%$ of the articular surface, a derotation osteotomy is necessary to maintain the joint in reduced position. Post-operatively, it is important to maintain the shoulder in adduction and the arm behind the sagittal axis of the body by strapping, to promote healing of the soft tissues and bone in normal reduced position.

\section{References}

1. Mclaughlin HL. Posterior dislocation of shoulder. J Bone Joint Surg 1952:30-A:581-90.

2. Swamy G. Schemitsch $\mathrm{EH}$. Humeral head fracture dislocation. J Trauma 1998:44::377-80.

3. Wadlington VR. Hendrix RW. Computed tomography of posterior dislocation of the shoulder. J Trauma 1992:32:1135.

4. Kirtland S. Resnik D. Sartoris DJ. Closed unreduced dislocation of glenohumeral joint. J Trauma 1998:28:162230 .

5. Wilson J. McKeever FM. Traumatic posterior dislocation of humerus. J Bone Joint Surg 1949:31-A:160-80.

6. Thomas MA. Posterior subacromial dislocation of the head of humerus. Am J Roentgenol 1937;32:767-73.

7. Cooper. A. On the dislocation of os humeri upon the dorsum scapulae, and upon the fractures about the shoulder joint. Guy's hospital report 1839:4:265-84.

8. Kaar TK . Worth M, Rockwood C. Missed dislocation of the humeral head. J Bone Joint Surg 1999;81-A;;08-10. 